\title{
Signals of a Quantum Universe
}

\author{
Daniel Green $\circledast^{1, *}$ and Rafael A. Porto $\circledast^{2, \dagger}$ \\ ${ }^{1}$ Department of Physics, University of California, San Diego, La Jolla, California 92093, USA \\ ${ }^{2}$ Deutsches Elektronen-Synchrotron DESY, Notkestrasse 85, 22607 Hamburg, Germany
}

(Received 27 February 2020; accepted 19 May 2020; published 24 June 2020)

\begin{abstract}
Structure in the Universe is widely believed to have originated from quantum fluctuations during an early epoch of accelerated expansion. Yet, the patterns we observe today do not distinguish between quantum or classical primordial fluctuations; current cosmological data are consistent with either possibility. We argue here that a detection of primordial non-Gaussianity can resolve the present situation, and provide a litmus test for the quantum origin of cosmic structure. Unlike in quantum mechanics, vacuum fluctuations cannot arise in classical theories and therefore long-range classical correlations must result from (real) particles in the initial state. Similarly to flat-space scattering processes, we show how basic principles require these particles to manifest themselves as poles in the $n$-point functions, in the so-called folded configurations. Following this observation, and assuming fluctuations are (i) correlated over large scales and (ii) generated by local evolution during an inflationary phase, we demonstrate that the absence of a pole in the folded limit of non-Gaussian correlators uniquely identifies the quantum vacuum as the initial state. In the same spirit as Bell's inequalities, we discuss how this can be circumvented if locality is abandoned.
\end{abstract}

DOI: 10.1103/PhysRevLett.124.251302

Introduction.-Cosmological observations strongly suggest that structure in the Universe originated from minute fluctuations present in the very early Universe, prior to the hot big bang [1-3]. A compelling possibility is that these density perturbations were produced through quantum mechanical zero-point fluctuations in the vacuum [4-8], and then were stretched over long distances by rapid accelerated expansion (inflation). In one brush, this idea unveils a beautiful connection between the largest structures in the cosmos and the fundamental laws of physics at the smallest scales. Yet, current data $[9,10]$ could equally be explained if inflation had stretched classical statistical fluctuations instead. In the same fashion as Bell's program back in the 1960s put quantum mechanics to the test [11], our goal here is to bring the quantum origin of the density fluctuations, realized in a majority of models, into a well-defined statement that can be confronted with future observations.

Unfortunately, one cannot simply perform experiments with the entire Universe. We only get to observe the one we inhabit, and only have access to an effectively classical probability distribution of fluctuations [12]. Classic tests of quantum mechanics, such as Bell's inequalities [11], cannot be directly applied in this case. As a result, despite a long history

Published by the American Physical Society under the terms of the Creative Commons Attribution 4.0 International license. Further distribution of this work must maintain attribution to the author(s) and the published article's title, journal citation, and DOI. Funded by SCOAP ${ }^{3}$. (see, e.g., Refs. [12-22]), until now there has been limited progress identifying observational connections between the quantum initial state and the classical universe we observe today. In this Letter we provide a testable prediction for the quantum nature of the primordial fluctuations.

A step toward a potential signature was suggested by Maldacena [23]. For a judiciously chosen model, the dynamics during inflation effectively performs a Bell-type measurement, storing the result in the final probability distribution. The proposal does not suggest a generic observational test; yet, although baroque, Maldacena's model is a proof of principle that the primordial fluctuations can remember their quantum origin. As we show here, the nonlinear local evolution of the density fluctuations can indeed store its quantum origin in the correlations observed at late times. Concretely, we will show how only quantum mechanics can produce the type of long-range correlations typical of the vacuum state, while classical fluctuations are necessarily produced by (highly excited) states with their own characteristic features. The basic picture, illustrated in Fig. 1, is the following: Non-Gaussian correlations in the quantum vacuum are associated with "particle creation". In contrast, due to locality, causal classical evolution must also include the decay of particles in the initial state. Hence, even though both vacuum and classical effects produce correlations on large scales at late times, the latter necessarily encode its distinctive physical origin, yielding distinguishable signatures from the case of quantum-vacuum fluctuations. In particular-in analogy with flat-space polology [24] — an associated pole must be present for classical 


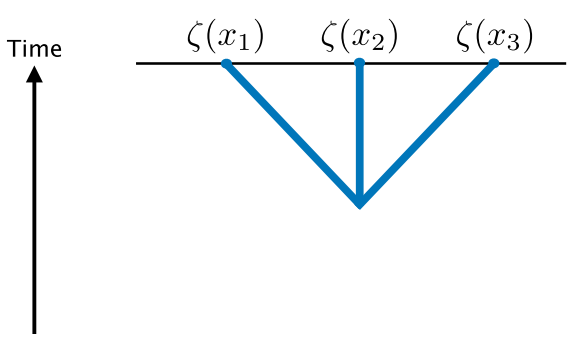

Quantum Vacuum

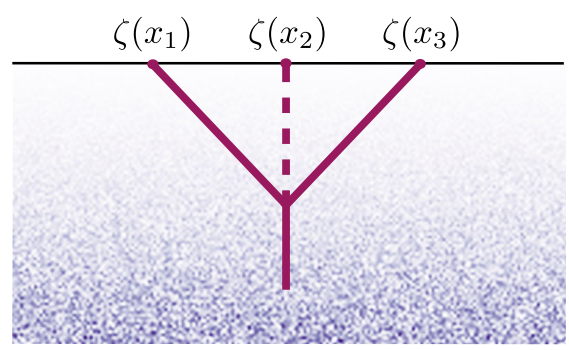

Classical

FIG. 1. Late-time observations measure correlations of the adiabatic density fluctuation $\zeta(x)$ produced from nonlinear time evolution in the early Universe. The particle's propagation is illustrated by the solid lines, while the dashed line represents the absence of the corresponding mode at late times. Left: Quantum-vacuum fluctuations arise as the correlated production of three particles due to nonlinear effects. This process would violate energy conservation in flat space, and thus produces no poles at physical momenta [31]. Right: Classical fluctuations only arise in a state containing physical particles, as local variations in the particle density; see, e.g., Refs. [25-30]. The nonlinear evolution that leads to net particle creation also allows for decays (or annihilation). These processes appear as poles at physical momenta.

$n$-point functions (beyond the power spectrum). Moreover, a width will also be generated, through dissipation [25-30], which effectively smooths these poles to produce a bump at physical momenta, as in particle colliders.

The existence of poles by itself may not be sufficient to show that classical physics is the culprit. For instance, quantum excited states can also develop the same pole structure [31]. Yet, we will demonstrate that the absence of this signature-in otherwise observable long-range nonGaussian correlations - can only be explained by quantum zero-point effects. In other words, in a classical framework consistent with locality, tampering with the analytic structure of the correlators in an attempt to remove the poles will unavoidably alter the structure at large scales, as expected from our intuition in flat space. On the other hand, longrange correlations - as those featured in the vacuum statemay be produced without the associated poles if locality is violated. We will illustrate the role of local causal evolution in an illuminating example.

Our analysis is also motivated by the practical issue of simulating a universe with non-Gaussian initial conditions. Typically, generating initial conditions with nonlocal correlations from a Gaussian map requires high-dimensional integration [32-34]. If these initial conditions were generated by local classical evolution instead, one could simply produce them via a Gaussian map evolved in time, and potentially speed up the simulations. However, as we show here, such a procedure - or any local evolution for that matter-will not accurately reproduce the non-Gaussian probability distribution obtained from quantum fluctuations. This result may also have some deeper relevance in quantum versus classical computing.

Cosmic quantumness: Gaussian fluctuations.-For concreteness, we will assume initial adiabatic density fluctuations $\zeta(x)$ arise from an effectively massless field during inflation. The background metric (ignoring slow-roll corrections) takes de Sitter's form: $d s^{2}=(-H \tau)^{-2}\left(-d \tau^{2}+\right.$ $d \mathbf{x}^{2}$ ), where physical $(t)$ and conformal $(\tau)$ times are related by $d t=a(\tau) d \tau=(-H \tau)^{-1} d \tau$, with $H$ the Hubble expansion parameter. We will define $\dot{f} \equiv \partial_{t} f=a^{-1} \partial_{\tau} f$ throughout. The modes of the density perturbations obey

$$
\zeta(\mathbf{x}, \tau)=\int \frac{d^{3} k}{(2 \pi)^{3}} e^{i \mathbf{k} \cdot \mathbf{x}}\left[a_{\mathbf{k}}^{\dagger} \zeta_{k}+a_{-\mathbf{k}} \zeta_{k}^{*}\right],
$$

where $\zeta_{k}=\Delta_{\zeta} k^{-3 / 2}(1-i k \tau) e^{i k \tau}$, with the normalization $\Delta_{\zeta}$ chosen to coincide with the observed amplitude of adiabatic fluctuations. Since the field is real, we have $\left(a_{\mathbf{k}}^{\dagger}\right)^{\dagger}=a_{-\mathbf{k}}$. The statistical differences arise once we compare quantum versus classical correlation functions.

Quantum.-The $a_{\mathbf{k}}^{\dagger}$ are creation operators in a Hilbert space, satisfying

$$
\left[a_{\mathbf{k}}^{\dagger}, a_{\mathbf{k}^{\prime}}\right]=\delta\left(\mathbf{k}-\mathbf{k}^{\prime}\right), \quad a_{\mathbf{k}}|0\rangle=0,
$$

which readily imply

$$
\left\langle 0\left|a_{\mathbf{k}^{\prime}} a_{\mathbf{k}}^{\dagger}\right| 0\right\rangle=\delta\left(\mathbf{k}-\mathbf{k}^{\prime}\right), \quad\left\langle 0\left|a_{\mathbf{k}}^{\dagger} a_{\mathbf{k}^{\prime}}\right| 0\right\rangle=0,
$$

in the vacuum state. In what follows, we will define $\langle 0|[\cdots]| 0\rangle \rightarrow\langle[\ldots]\rangle_{q}$ for convenience.

Classical.-The $a_{\mathbf{k}}^{\dagger}$ are stochastic parameters, which obey the following statistical properties:

$$
\left\langle a_{\mathbf{k}}^{\dagger} a_{\mathbf{k}^{\prime}}\right\rangle_{c}=\frac{1}{2} \delta\left(\mathbf{k}-\mathbf{k}^{\prime}\right)=\left\langle a_{\mathbf{k}^{\prime}} a_{\mathbf{k}}^{\dagger}\right\rangle_{c},
$$

as an ensemble average. Notice that the second equality is only valid for classical fluctuations, since it implies that the stochastic parameters must commute.

By construction, both classical and quantum-vacuum fluctuations give rise to the same equal-time correlation functions in the absence of interactions. Therefore, measurements of the power spectrum alone are not sufficient to distinguish between them. On the other hand, for unequal times the quantum and 
classical two-point functions do not agree, reflecting the nonzero commutator, $[\zeta(x, \tau), \dot{\zeta}(x, \tau)] \neq 0$, in the quantum theory. This distinction plays a key role when interactions are present.

Non-Gaussianity.-In order to gain intuition, we will consider an illustrative example with the interaction Hamiltonian $H_{\text {int }}=-(\lambda / 3 !) \dot{\zeta}^{3}$. This choice will allow us to perform explicit computations without losing generality. Our conclusions will be rooted in well-established principles, and therefore do not depend on the type of interaction as long as it respects locality.

Quantum.-The standard (in-in) calculation [35] in the vacuum state yields [36] $\left(\left|\mathbf{k}_{i}\right|=k_{i}\right)$

$$
\left\langle\zeta_{\mathbf{k}_{1}} \zeta_{\mathbf{k}_{2}} \zeta_{\mathbf{k}_{3}}\right\rangle_{q}^{\prime}=\frac{4 \lambda H^{2} \Delta_{\zeta}^{6}}{\left(k_{1}+k_{2}+k_{3}\right)^{3} k_{1} k_{2} k_{3}},
$$

up to the momentum-conserving $\delta$ function, which is denoted by the primed brackets \langle\rangle$^{\prime}$. Notice, for $k_{i} \neq 0$, we only have a pole in the total energy: $k_{t} \equiv k_{1}+k_{2}+k_{3}$. This is due to the fact that, for cosmological (in-in) correlators, the would-be energy-conserving $\delta$ function becomes a factor of $1 / k_{t}^{n}$, for non-negative integer $n$. Via analytic continuation, as $k_{t} \rightarrow 0$, the residue of this pole is intimately connected to the flat-space $S$ matrix, with the order of the pole $(n=3$ in this case) related to the number of derivatives at the local interaction [37-42]. In the quantum vacuum, the correlation is produced by the creation of three (virtual) particles $(0 \rightarrow 3)$, which are subsequently measured at later times (see Fig. 1). The uncertainty principle in an expanding universe permits aminimal amount of - violation of energy conservation, $\Delta t \sim H^{-1}$, which is forbidden classically [31]. As expected, since there are no real particles to scatter in the vacuum, there are no other processes allowed nor poles at physical momenta.

Classical.-We determine the bispectrum by solving the classical equations of motion perturbatively. Using the Green's function, obeying

$\partial_{\tau^{\prime}} G\left(\tau, \tau^{\prime}\right)=2 \Delta_{\zeta}^{2}\left[\frac{\tau^{\prime}}{k} \sin \left[k\left(\tau-\tau^{\prime}\right)\right]-\tau \tau^{\prime} \cos \left[k\left(\tau-\tau^{\prime}\right)\right]\right]$,

we find at first order in $\lambda$,

$$
\begin{aligned}
\zeta_{\mathbf{k}}^{(2)}(\tau)= & \lambda \int \frac{d \tau^{\prime} d^{3} p}{(2 \pi)^{3}}\left(-H \tau^{\prime}\right)^{-1} \\
& \times\left[\partial_{\tau^{\prime}} G_{\mathbf{k}}\left(\tau, \tau^{\prime}\right)\right] \partial_{\tau^{\prime}} \zeta_{\mathbf{p}}^{(1)}\left(\tau^{\prime}\right) \partial_{\tau^{\prime}} \zeta_{\mathbf{k}-\mathbf{p}}^{(1)}\left(\tau^{\prime}\right)
\end{aligned}
$$

where $\zeta^{(1)}$ is the Gaussian field. Using $\zeta_{\mathbf{k}} \approx \zeta_{\mathbf{k}}^{(1)}+\zeta_{\mathbf{k}}^{(2)}$, the leading contribution to the bispectrum at $\tau=0$ becomes

$$
\begin{aligned}
\left\langle\zeta_{\mathbf{k}_{1}} \zeta_{\mathbf{k}_{2}} \zeta_{\mathbf{k}_{3}}\right\rangle_{c}^{\prime}= & \frac{2 \lambda H^{2} \Delta_{\zeta}^{6}}{3 k_{1} k_{2} k_{3}}\left[\frac{3}{k_{t}^{3}}+\frac{1}{\left(k_{1}+k_{2}-k_{3}\right)^{3}}\right. \\
& \left.+\frac{1}{\left(k_{1}-k_{2}+k_{3}\right)^{3}}+\frac{1}{\left(k_{2}-k_{1}+k_{3}\right)^{3}}\right]
\end{aligned}
$$

As much anticipated, there are poles at physical momenta, in addition to the one at $k_{t}=0$. These poles are due to classical fluctuations of physical (real) particles in the initial state, which (nonlinearly) interact to produce longrange non-Gaussian correlations. For instance, physical particles can decay (annihilate) via on-shell $1 \rightarrow 2(2 \rightarrow 1)$ processes, and therefore are associated with the poles in the so-called folded limit [43], where $k_{1} \rightarrow k_{2}+k_{3}$ and permutations thereof (see Fig. 1).

Signatures of quantum origin.-The above example illustrates a general property of (in-in) inflationary correlation functions: poles at physical momenta arise from the scattering of real particles present in the initial state. For quantum-vacuum fluctuations there are no real particles, only virtual, yet the poles are still present (by analytic continuation) at negative energies. This is more than just an isolated result mimicking our flat-space intuition. In fact, notice that the overall coefficients of the poles, either in the quantum Eq. (5) or classical correlation Eq. (9) are related, and ultimately linked to the scattering amplitude in the flatspace limit [37-42]. Hence, following basic principles, causality guarantees that any process that creates (real) particles at local events is necessarily accompanied by physical poles in the correlation functions [44]. The specific form of the interaction controls the resulting polynomial in momentum and/or time dependence, and hence only affects the residue of the poles. Let us emphasize that this is an unavoidable conclusion, which does not depend on the form of the (local) interaction. As a consequence, since there are no vacuum fluctuations in classical mechanics, quantum mechanics is the only way we can guarantee a non-Gaussian signal without violations of causality or locality, while avoiding the existence of poles at physical momenta.

As usual [24], decay processes will introduce a finite width which softens the behavior in the folded limit. However, unlike the drift toward the complex plane found in flat space, for (in-in) correlators in an expanding universe the poles move away from the "mass shell" but remain real. While the existence of a width usually happens at higher orders in perturbation theory, models with strong dissipation will exhibit this softening already at tree level.

Classical nonlocalities. - A crucial aspect of Bell's inequalities is that they may be circumvented by nonlocal theories with hidden variables at the classical level $[11,45,46]$. Similarly, locality plays a key role in inferring the quantum nature of the cosmological signal. For our purposes here, it will be sufficient to find an example of a theory which reproduces the same correlators as the 
quantum vacuum, but violates locality. At the same time, we will show that enforcing local causal evolution, while attempting to remove the poles, also alters the type of longrange correlations expected in the vacuum state.

Hidden variables.-For illustrative purposes, we consider a complex scalar field, which may be decomposed as

$\phi_{\mathbf{k}}(\tau)=\frac{\Delta_{\phi}}{k^{3 / 2}}\left[a_{\mathbf{k}}^{\dagger}(1-i k \tau) e^{i k \tau}+b_{-\mathbf{k}}(1+i k \tau) e^{-i k \tau}\right]$,

obeying classical Gaussian statistics,

$$
\left\langle a_{\mathbf{k}}^{\dagger} a_{\mathbf{k}^{\prime}}\right\rangle_{c}=\left\langle a_{\mathbf{k}^{\prime}} a_{\mathbf{k}}^{\dagger}\right\rangle_{c}=\delta\left(\mathbf{k}-\mathbf{k}^{\prime}\right), \quad\left\langle b_{\mathbf{k}} b_{\mathbf{k}^{\prime}}^{\dagger}\right\rangle_{c}=0 .
$$

Let us assume now the existence of a Lagrangian such that the following modified Green's function,

$$
\partial_{\tau^{\prime}} G_{\mathbf{k}}\left(\tau \rightarrow 0, \tau^{\prime}\right) \rightarrow G_{\mathbf{k}}^{\mathrm{eff}}\left(\tau \rightarrow 0, \tau^{\prime}\right)=\frac{1}{k} e^{-i k \tau^{\prime}},
$$

applies in the $\tau \rightarrow 0$ limit. Notice that it includes only positive frequency modes. We will not specify the nature of the interaction leading to the above properties, and therefore we treat it as a hidden-variable theory. Using Eq. (7) with $\zeta \rightarrow \phi$ and $\lambda \rightarrow \lambda_{\phi}$, we find

$\phi_{\mathbf{k}}(\tau \rightarrow 0)=\frac{i}{3} \lambda_{\phi} \int \frac{d^{3} p}{(2 \pi)^{3}} \int d \tau^{\prime} \frac{1}{k} e^{-i k \tau^{\prime}} \dot{\phi}_{\mathbf{p}}^{*}\left(\tau^{\prime}\right) \dot{\phi}_{\mathbf{k}-\mathbf{p}}^{*}\left(\tau^{\prime}\right)$.

From this expression we can calculate the bispectrum as usual, yielding

$$
\left\langle\phi_{\mathbf{k}_{1}} \phi_{\mathbf{k}_{2}} \phi_{\mathbf{k}_{3}}\right\rangle^{\prime}=\frac{2 \lambda_{\phi} H^{-1} \Delta_{\phi}^{6}}{\left(k_{1}+k_{2}+k_{3}\right)^{3} k_{1} k_{2} k_{3}} .
$$

Since $\phi$ is real at late times, it can be converted into density fluctuations after inflation, e.g., $\phi(\tau \rightarrow 0) \approx \zeta$. Up to an overall constant, the result reproduces the same statistical map for the quantum vacuum in Eq. (5) (up to higher order effects which are not relevant here). This theory, however, is nonlocal as it can be seen directly from Eq. (11). In particular, locality demands that the Green's function in coordinate space must vanish outside of the light cone. Yet, we have $G_{\mathbf{k}}^{\text {eff }}\left(\tau^{\prime} \rightarrow 0\right) \simeq k^{-1}$, resembling the Coulomb potential, which is nonzero everywhere in space. As a consequence, this theory propagates information instantaneously everywhere in space-time. This is not surprising as causality in a relativistic theory demands the presence of a negative frequency mode ("antiparticle") [47], which is precisely what gives rise to the poles at physical momenta.

Conclusions and outlook.-The origin of structure as a result of vacuum fluctuations is a purely quantum mechanical phenomena, for classical effects can only arise when states contain (many) physical particles. Moreover, due to causality, nonlinear interactions that allow for the creation of particles must be accompanied by processes in which particles are also able to decay. While the creation of (virtual) particles is allowed, decays are forbidden in vacuum, which gives rise to a dramatic difference in the types of non-Gaussian correlations arising in classical versus quantum-vacuum fluctuations.

The distinction between the two results, as well as the role of locality, is also manifest in the manipulations involved in the derivation of the three-point function. In general, one can show that the difference between the quantum-vacuum and classical computation may be written as

$$
\begin{array}{r}
\left\langle\zeta\left(\mathbf{x}_{1}, \tau\right) \zeta\left(\mathbf{x}_{2}, \tau\right) \zeta\left(\mathbf{x}_{3}, \tau\right)\right\rangle_{q}-\left\langle\zeta\left(\mathbf{x}_{1}, \tau\right) \zeta\left(\mathbf{x}_{2}, \tau\right) \zeta\left(\mathbf{x}_{3}, \tau\right)\right\rangle_{c} \\
=\frac{i \lambda}{24} \sum_{\sigma} \int_{-\infty}^{\tau} d^{3} \mathbf{x}^{\prime} d \tau^{\prime} a^{4}\left(\tau^{\prime}\right)\left[\zeta\left(\mathbf{x}_{1}, \tau\right), \hat{D}_{\sigma(1)} \zeta\left(\mathbf{x}^{\prime}, \tau^{\prime}\right)\right] \\
\quad \times\left[\zeta\left(\mathbf{x}_{2}, \tau\right), \hat{D}_{\sigma(2)} \zeta\left(\mathbf{x}^{\prime}, \tau^{\prime}\right)\right]\left[\zeta\left(\mathbf{x}_{3}, \tau\right), \hat{D}_{\sigma(3)} \zeta\left(\mathbf{x}^{\prime}, \tau^{\prime}\right)\right],
\end{array}
$$

where $\hat{D}_{\ell=1,2,3}$ are local differential operators that characterize the type of interaction(s) and $\sigma$ is a permutation. The above expression neatly illustrates the link between late time measurements in a quantum state and Bell-type correlations at an earlier time, which are encoded in the (nonvanishing) commutators. Moreover, because of causality, the commutators vanish at spacelike separation. Therefore, the above difference is built up from interactions in the overlap between the past light cones of the points $\mathbf{x}_{1}, \mathbf{x}_{2}$, and $\mathbf{x}_{3}$. This implies that the information encoded in the correlators cannot be modified by local operations at late times. For quantum-vacuum fluctuations, the absence of a pole in the folded limit of the bispectrum thus becomes a unique signature of local causal evolution.

Limits of various $n$-point functions have been known to encode important physical information; see, e.g., Refs. [28,31,39,48-52]. An enhanced "soft limit" (with soft internal or external momenta) is due to additional (light) fields, while the folded limit is enhanced for excited states. Yet, as we have demonstrated here, the absence of an enhancement in folded configurations cannot occur with classical fluctuations, which would provide-barring violations of locality-striking evidence for the quantum origin of structure in the Universe. Although current observations are consistent with a Gaussian spectrum, surveys of increasing volume and sensitivity will continue the search for non-Gaussianity [53]. A true pole in the folded limit of the $n$-point functions would have diverging signal-to-noise ratio [43]. While the nonzero width of physical particles will make the signal-to-noise ratio finite, it is detectable nonetheless. However, enhanced dissipation may increase the width, thus reducing the signal-to-noise ratio in the folded limit. At the same time, dissipation also increases the amplitude of the overall non-Gaussianty $[28,30,54]$, which is presently constrained by data [10]. 
Hence, as a matter of principle, it is possible (but potentially challenging) to distinguish between the spectrum of classical and quantum fluctuations. Moreover, as we have emphasized, the analytic structure of their respective shapes is clearly distinct, which suggests an analysis in position space might provide a more stringent test, perhaps along the line discussed in Ref. [55].

Finally, although we restricted ourselves to the case of density fluctuations, our results are also relevant for primordial gravitational waves [54,56,57]. A signal could be the consequence of either quantum or classical fluctuations. However, the latter is correlated with measurable effects also in the density perturbations [54,58]. As we have shown, the shape of the associated non-Gaussianity will ultimately reveal its cosmic origin.

We are grateful to Daniel Baumann, Roland de Putter, Raphael Flauger, Juan Maldacena, John McGreevy, Alec Ridgway, Uroš Seljak, Benjamin Wallisch, and Matias Zaldarriaga for helpful discussions. We would like to thank also the participants of the "Amplitudes meet Cosmology" workshop for useful conversations. D. G. is supported by the U.S. Department of Energy under Grant No. DESC0019035. R. A. P. acknowledges financial support from the ERC Consolidator Grant "Precision Gravity: From the LHC to LISA" provided by the European Research Council (ERC) under the European Union's H2020 research and innovation programme (Grant Agreement No. 817791), as well as from the Deutsche Forschungsgemeinschaft (DFG, German Research Foundation) under Germany's Excellence Strategy (EXC 2121) "Quantum Universe" (390833306).

*drgreen@physics.ucsd.edu †rafael.porto@desy.de

[1] W. Hu, D. N. Spergel, and M. J. White, Distinguishing causal seeds from inflation, Phys. Rev. D 55, 3288 (1997).

[2] D. N. Spergel and M. Zaldarriaga, CMB Polarization as a Direct Test of Inflation, Phys. Rev. Lett. 79, 2180 (1997).

[3] S. Dodelson, Coherent phase argument for inflation, AIP Conf. Proc. 689, 184 (2003).

[4] V. F. Mukhanov and G. V. Chibisov, Quantum fluctuations and a nonsingular universe, Pis'ma Zh. Eksp. Teor. Fiz. 33, 549 (1981) [JETP Lett. 33, 532 (1981)], http://www .jetpletters.ac.ru/ps/1510/article_23079.shtml.

[5] S. W. Hawking, The development of irregularities in a single bubble inflationary universe, Phys. Lett. 115B, 295 (1982).

[6] A. H. Guth and S. Y. Pi, Fluctuations in the New Inflationary Universe, Phys. Rev. Lett. 49, 1110 (1982).

[7] A. A. Starobinsky, Dynamics of phase transition in the new inflationary universe scenario and generation of perturbations, Phys. Lett. 117B, 175 (1982).

[8] J. M. Bardeen, P. J. Steinhardt, and M. S. Turner, Spontaneous creation of almost scale-free density perturbations in an inflationary universe, Phys. Rev. D 28, 679 (1983).
[9] Y. Akrami et al. (Planck Collaboration), Planck 2018 results. X. Constraints on inflation, Astrophys. Space Sci. 364, 69 (2019).

[10] Y. Akrami et al. (Planck Collaboration), Planck 2018 results. IX. Constraints on primordial non-Gaussianity, arXiv:1905.05697.

[11] J. S. Bell, On the Einstein-Podolsky-Rosen paradox, Phys. Phys. Fiz. 1, 195 (1964).

[12] L. P. Grishchuk and Yu. V. Sidorov, Squeezed quantum states of relic gravitons and primordial density fluctuations, Phys. Rev. D 42, 3413 (1990).

[13] A. A. Starobinsky, Stochastic de Sitter (inflationary) stage in the early Universe, Lect. Notes Phys. 246, 107 (1986).

[14] D. Campo and R. Parentani, Inflationary spectra and violations of Bell inequalities, Phys. Rev. D 74, 025001 (2006).

[15] E. A. Lim, Quantum information of cosmological correlations, Phys. Rev. D 91, 083522 (2015).

[16] J. Martin and V. Vennin, Quantum discord of cosmic inflation: Can we show that $\mathrm{CMB}$ anisotropies are of quantum-mechanical origin?, Phys. Rev. D 93, 023505 (2016).

[17] S. Goldstein, W. Struyve, and R. Tumulka, The Bohmian approach to the problems of cosmological quantum fluctuations, arXiv:1508.01017.

[18] E. Nelson, Quantum decoherence during inflation from gravitational nonlinearities, J. Cosmol. Astropart. Phys. 03 (2016) 022.

[19] S. Choudhury, S. Panda, and R. Singh, Bell violation in the sky, Eur. Phys. J. C 77, 60 (2017).

[20] J. Martin and V. Vennin, Obstructions to Bell CMB experiments, Phys. Rev. D 96, 063501 (2017).

[21] S. Shandera, N. Agarwal, and A. Kamal, Open quantum cosmological system, Phys. Rev. D 98, 083535 (2018).

[22] R. de Putter and O. Doré, In search of an observational quantum signature of the primordial perturbations in slowroll and ultra slow-roll inflation, Phys. Rev. D 101, 043511 (2020).

[23] J. Maldacena, A model with cosmological Bell inequalities, Fortschr. Phys. 64, 10 (2016).

[24] S. Weinberg, The Quantum Theory of Fields. Vol. 1: Foundations (Cambridge University Press, Cambridge, England, 2005).

[25] A. Berera, Warm Inflation, Phys. Rev. Lett. 75, 3218 (1995).

[26] A. Berera, M. Gleiser, and R. O. Ramos, A First Principles Warm Inflation Model that Solves the Cosmological Horizon/Flatness Problems, Phys. Rev. Lett. 83, 264 (1999).

[27] D. Green, B. Horn, L. Senatore, and E. Silverstein, Trapped inflation, Phys. Rev. D 80, 063533 (2009).

[28] D. Lopez Nacir, R. A. Porto, L. Senatore, and M. Zaldarriaga, Dissipative effects in the effective field theory of inflation, J. High Energy Phys. 01 (2012) 075.

[29] D. Lopez Nacir, R. A. Porto, and M. Zaldarriaga, The consistency condition for the three-point function in dissipative single-clock inflation, J. Cosmol. Astropart. Phys. 09 (2012) 004.

[30] G. J. Turiaci and M. Zaldarriaga, Non-Gaussianities in dissipative EFT of inflation coupled to a fluid, arXiv: 1310.4531. 
[31] R. Flauger, D. Green, and R. A. Porto, On squeezed limits in single-field inflation. Part I, J. Cosmol. Astropart. Phys. 08 (2013) 032.

[32] K. M. Smith and M. Zaldarriaga, Algorithms for bispectra: Forecasting, optimal analysis, and simulation, Mon. Not. R. Astron. Soc. 417, 2 (2011).

[33] F. Schmidt and M. Kamionkowski, Halo clustering with non-local non-Gaussianity, Phys. Rev. D 82, 103002 (2010).

[34] R. Scoccimarro, L. Hui, M. Manera, and K. C. Chan, Largescale bias and efficient generation of initial conditions for non-local primordial non-Gaussianity, Phys. Rev. D 85, 083002 (2012).

[35] S. Weinberg, Quantum contributions to cosmological correlations, Phys. Rev. D 72, 043514 (2005).

[36] See Supplemental Material at http://link.aps.org/ supplemental/10.1103/PhysRevLett.124.251302 for detailed derivations of a number of important equations in this Letter.

[37] J. M. Maldacena and G. L. Pimentel, On graviton nonGaussianities during inflation, J. High Energy Phys. 09 (2011) 045.

[38] S. Raju, New recursion relations and a flat space limit for AdS/CFT correlators, Phys. Rev. D 85, 126009 (2012).

[39] N. Arkani-Hamed and J. Maldacena, Cosmological collider physics, arXiv:1503.08043.

[40] N. Arkani-Hamed, D. Baumann, H. Lee, and G. L. Pimentel, The cosmological bootstrap: Inflationary correlators from symmetries and singularities, J. High Energy Phys. 04 (2020) 105.

[41] N. Arkani-Hamed and P. Benincasa, On the emergence of Lorentz invariance and unitarity from the scattering facet of cosmological polytopes, arXiv:1811.01125.

[42] P. Benincasa, From the flat-space S-matrix to the wavefunction of the Universe, arXiv:1811.02515.

[43] D. Babich, P. Creminelli, and M. Zaldarriaga, The shape of non-Gaussianities, J. Cosmol. Astropart. Phys. 08 (2004) 009.

[44] H. Lehmann, K. Symanzik, and W. Zimmermann, On the formulation of quantized field theories, Nuovo Cimento $\mathbf{1}$, 205 (1955).
[45] D. Bohm, A suggested interpretation of the quantum theory in terms of hidden variables. 1, Phys. Rev. 85, 166 (1952).

[46] D. Bohm, A suggested interpretation of the quantum theory in terms of hidden variables. II, Phys. Rev. 85, 180 (1952).

[47] R. P. Feynman, The reason for antiparticles, in Elementary Particles and the Laws of Physics: The 1986 Dirac Memorial Lectures (Cambridge University Press, Cambridge, England, 1987), pp. 1-60.

[48] J. M. Maldacena, Non-Gaussian features of primordial fluctuations in single field inflationary models, J. High Energy Phys. 05 (2003) 013.

[49] P. Creminelli and M. Zaldarriaga, Single field consistency relation for the 3-point function, J. Cosmol. Astropart. Phys. 10 (2004) 006.

[50] V. Assassi, D. Baumann, and D. Green, On soft limits of inflationary correlation functions, J. Cosmol. Astropart. Phys. 11 (2012) 047.

[51] W. D. Goldberger, L. Hui, and A. Nicolis, One-particleirreducible consistency relations for cosmological perturbations, Phys. Rev. D 87, 103520 (2013).

[52] R. Holman and A. J. Tolley, Enhanced non-Gaussianity from excited initial states, J. Cosmol. Astropart. Phys. 05 (2008) 001.

[53] P. D. Meerburg et al., Primordial non-Gaussianity, arXiv: 1903.04409.

[54] R. A. Porto, Gravitational waves and the (quantum) nature of the primordial seed, Int. J. Mod. Phys. D 23, 1441005 (2014).

[55] M. Münchmeyer and K. M. Smith, Higher $N$-point function data analysis techniques for heavy particle production and WMAP results, Phys. Rev. D 100, 123511 (2019).

[56] L. Senatore, E. Silverstein, and M. Zaldarriaga, New sources of gravitational waves during inflation, J. Cosmol. Astropart. Phys. 08 (2014) 016.

[57] S. Shandera et al., Probing the origin of our Universe through cosmic microwave background constraints on gravitational waves, arXiv:1903.04700.

[58] M. Mirbabayi, L. Senatore, E. Silverstein, and M. Zaldarriaga, Gravitational waves and the scale of inflation, Phys. Rev. D 91, 063518 (2015). 